\title{
Patients' views of physical activity as treatment for depression: a qualitative study
}

\author{
Aidan Searle, Michael Calnan, Glyn Lewis, John Campbell,
} Adrian Taylor and Katrina Turner

\begin{abstract}
Background

Clinical guidance recommends physical activity to manage patients with persistent subthreshold depressive symptoms or mild-to-moderate depression. However, little is known regarding the acceptability of physical activity as a treatment for depression from patients' perspective.
\end{abstract}

Aim

To explore patients' views of physical activity for the treatment of depression in the context of primary care.

Design of study

In-depth interviews were held with 33 participants taking part in a randomised controlled trial assessing the effectiveness of physical activity for the management of depression.

Setting

Primary care.

Results

Most participants perceived physical activity to be an acceptable treatment for depression. The mechanisms by which physical activity could enhance mood were attributed to a number of subjective benefits including biochemical pathways, providing a source of distraction from negative thoughts, and a sense of purpose. Participants who expressed a belief that their depression was caused by biochemical mechanisms reported activity that 'raised the heartbeat' as most beneficial, while those who believed depression was situational in origin tended to state the benefits of lessaerobic activities, such as walking. Many participants reported low motivation and a lack of confidence as barriers to undertaking physical activity. These patients suggested that medication could be helpful for initiating and maintaining activity.

Conclusion

Patients view physical activity as an effective treatment for depression. However, they vary in their views about how physical activity might impact on depression, what intensity and form of activity is necessary to enhance mood, and the barriers to undertaking activity. This variation suggests the need for GPs to elicit patients' views on physical activity as a treatment, and offer interventions that are tailored to the needs and expectations of individual patients.

Keywords

antidepressants; depression; guidelines; physical activity; primary health care; qualitative research.

\section{INTRODUCTION}

There is growing evidence that physical activity can be an effective treatment for depression. ${ }^{1}$ The National Institute for Health and Clinical Excellence (NICE) recommends that GPs consider offering a structured group-based physical activity programme to patients with persistent subthreshold depressive symptoms or mild-to-moderate depression. ${ }^{2}$ At present, patients with depression may be offered an exercise referral scheme, either as an adjunct to medication or as an alternative treatment option. ${ }^{3}$ In the UK, $80 \%$ of GPs who have access to exercise referral schemes use them in the treatment of their depressed patients. ${ }^{4}$ However, the take-up and drop-out rates for patients referred to exercise schemes for their general health is highly variable, due to low motivation, lack of social support, and time constraints., ${ }^{5,6}$ Such barriers to engaging in physical activity are likely to be compounded among patients with depression. ${ }^{7}$

Depression is set to become the second largest

A Searle, BSc(Hons), $M S c, P h D$, research associate; G Lewis, MSc (Lond), PhD, MB BS, professor of psychiatric epidemiology; K Turner, PhD, lecturer, Academic Unit of Psychiatry, School of Social and Community Medicine, University of Bristol, Bristol. M Calnan, PhD, professor of medical sociology, School of Social Policy, Sociology and Social Research, University of Kent, Canterbury. J Campbell, MD, MRCGP, associate dean and professor of general practice and primary care, Primary Care Research Group, Peninsula Medical School; A Taylor, PhD, professor of sport and exercise psychology, School of Sport and Health Sciences, University of Exeter, Exeter.

Address for correspondence

Dr Aidan Searle, Academic Unit of Primary Care, School of Social and Community Medicine, University of Bristol, 39 Whatley Road, Bristol, BS8 2PS.

E-mail: A.J.Searle@bristol.ac.uk

Submitted: 9 July 2010; Editor's response: 20 August 2010; final acceptance: 18 October 2010.

(C) British Journal of General Practice

This is the full-length article (published online 28 Mar 2011) of an abridged version published in print. Cite this article as: Br J Gen Pract 2011; DOI: 10.3399/bjgp1 1X567054. 
cause of global disability by $2020,{ }^{8}$ and represents a significant challenge to primary care services in the UK. ${ }^{9}$ There has also been a dramatic increase in antidepressant medication prescribing, and growing concern regarding the overtreatment of human distress and the safety of antidepressants..$^{10}$ In addition, the cost of prescribing antidepressant medication suggests there is a need to establish the most appropriate balance between medication and nonpharmacological treatments for depression. ${ }^{11}$

Qualitative evidence suggests that GPs' decisions to prescribe antidepressants are shaped by organisational constraints, such as time, access to alternative management options, cost, and patient attitudes. $^{12}$ There is also evidence that when antidepressants are prescribed, adherence is often poor. ${ }^{13}$ However, there has been no qualitative exploration of how people with depression, who have not been referred to exercise referral schemes, view physical activity for managing mental health.

Faulkner and Biddle explored the views of patients with a diagnosis of depression referred to exercise referral schemes. ${ }^{14}$ Their case-study approach highlighted that the potential for physical activity to treat depression was idiosyncratic in terms of both its process and its efficacy. More recent work has explored the views and use of physical activity for mental health in a non-clinical sample, where it was found that patients use physical activity in a 'selfregulatory' way to manage symptoms of depression. ${ }^{15}$ The present article reports on a qualitative exploration of patients' views of physical activity as a potential treatment for depression in the context primary care.

\section{METHOD}

\section{Study design and setting}

Interviews were held with participants taking part in TREAD (Trial of Exercise And Depression), an ongoing pragmatic randomised controlled trial of facilitated physical activity plus usual care, versus usual care only for the treatment of depression. ${ }^{16}$ Participants were recruited from GP practices from Bristol and Devon Primary Care Trusts.

Participants in the usual-care arm attended regular appointments with their GP, and some were in receipt of antidepressant medication and or/counselling. The facilitated physical activity intervention was tailored on an individual basis and comprised behavioural change and motivational interviewing techniques delivered through face-to-face meetings with a facilitator and regular telephone contact.

All patients aged 18-69 years who had either recently started antidepressants (within 4 weeks of their assessment and following an antidepressant-free period of at least 1 month), or who were not currently on antidepressants but had recently consulted their GP

\section{How this fits in}

Physical activity has been shown to be effective in treating depression.

However, little is known about how patients regard physical activity as a treatment for depression. In this study it was found that many patients view physical activity as an effective and acceptable treatment for depression. However, the breadth of patients' views and experience of physical activity suggests that offering structured, group-based programmes as recommended in current clinical guidelines may not be practical or sufficient for many patients.

for depression and met the International Classification of Diseases (ICD-10) criteria determined by the Clinical Interview Schedule Revised (CIS-R), ${ }^{17}$ were recruited to the trial by direct GP referral or electronic searches of GP records. Individuals referred with a history of psychosis, bipolar disorder, major alcohol or substance abuse, and contraindications to physical activity, or who were pregnant or breastfeeding were excluded from participation.

All trial participants received an information sheet prior to consenting to take part in the trial. This sheet stated there is evidence to suggest physical activity may be helpful in the treatment of depression but that this evidence is limited. The sheet also stated that trial participants may be interviewed about their views on physical activity as a treatment for depression.

Trial participants were invited for interview once they had completed their primary outcome measures, which included activity logs and a measure of depression $^{18}$ for the trial at 4 months postrandomisation. Participants were purposefully sampled from both trial centres and from both arms of the trial. This was a pragmatic trial and thus it was not possible to blind participants to their treatment allocation, as it would be obvious which arm of the trial they were in. Within this sampling approach, the study aimed for maximum variation in relation to age, sex, practice location (urban, suburban, and rural), severity of depression, and self-reported activity level at baseline.

Severity of depression was categorised at baseline by CIS-R score; $\leq 25$ (mild), 26-33 (moderate), $\geq 34$ (severe). Activity level was categorised as the number of days per week that the participant reported engaging in moderate (for example, walking, easy swimming) or vigorous (for example, squash, running) physical activity (low $=0-1$, medium $=2-3$, high $=$ 4-7 days).

\section{Data collection}

Forty-five trial participants were approached for interview. Of these, 33 participants were interviewed by between March and November 2009 (Table 1). Those who declined an interview typically gave time restraints as a reason for non-participation.

Twenty-eight participants were interviewed in their 
Table 1. Patient characteristics $(n=33)$

\begin{tabular}{|c|c|c|c|}
\hline & All patients, $n(\%)^{\mathrm{a}}$ & Usual care, $n$ & Intervention, $n$ \\
\hline Age range, years & $19-69$ & $21-65$ & $19-69$ \\
\hline Male & $14(42.4)$ & 6 & 8 \\
\hline Female & $19(57.5)$ & 8 & 11 \\
\hline \multicolumn{4}{|l|}{ Study site } \\
\hline Bristol & $16(48.5)$ & 6 & 10 \\
\hline Exeter & $17(51.5)$ & 9 & 8 \\
\hline \multicolumn{4}{|l|}{ Marital status } \\
\hline Married/cohabiting & $18(54.5)$ & 9 & 9 \\
\hline Single & $9(27.3)$ & 5 & 4 \\
\hline Separated/divorced & $4(12.1)$ & 3 & 1 \\
\hline Widowed & $2(6.1)$ & 0 & 2 \\
\hline \multicolumn{4}{|l|}{ Employment status } \\
\hline Employed full-time & $14(42.4)$ & 6 & 8 \\
\hline Employed part-time & $5(15.2)$ & 3 & 2 \\
\hline Unemployed & $4(12.1)$ & 1 & 3 \\
\hline Retired & $4(12.1)$ & 1 & 3 \\
\hline Permanent sick/full-time carer & $4(12.1)$ & 2 & 2 \\
\hline Training/education & $2(6.1)$ & 0 & 2 \\
\hline \multicolumn{4}{|l|}{ Educational attainment } \\
\hline Higher degree & $2(6.0)$ & 1 & 1 \\
\hline Degree & $10(30.3)$ & 3 & 7 \\
\hline Diploma & $5(15.2)$ & 4 & 1 \\
\hline A level & $19(57.6)$ & 10 & 9 \\
\hline GCSE/O level & $23(69.7)$ & 10 & 13 \\
\hline Other & $3(9.1)$ & 1 & 2 \\
\hline None & $4(12.1)$ & 2 & 2 \\
\hline \multicolumn{4}{|l|}{ Housing status } \\
\hline Home owner & $19(57.6)$ & 7 & 12 \\
\hline Tenant & 11 (33.3) & 7 & 4 \\
\hline Living with relative & $3(9.1)$ & 0 & 3 \\
\hline \multicolumn{4}{|l|}{ Ethnicity } \\
\hline White & $31(94.0)$ & 12 & 19 \\
\hline Asian & $1(3.0)$ & 1 & 0 \\
\hline Mixed & $1(3.0)$ & 0 & 1 \\
\hline Current antidepressant & $22(66.7)$ & 1 & 21 \\
\hline Past depression & $23(69.7)$ & 10 & 13 \\
\hline Physical comorbidity & $16(48.5)$ & 8 & 8 \\
\hline Hypertension/heart disease & $6(18.2)$ & 4 & 2 \\
\hline Rheumatoid arthritis/fibromyalgia & $2(6.1)$ & 1 & 1 \\
\hline Cancer & $2(6.1)$ & 1 & 1 \\
\hline Back pain & $2(6.1)$ & 1 & 1 \\
\hline Stroke & $1(3.0)$ & 1 & 0 \\
\hline Diabetes & $1(3.0)$ & 0 & 1 \\
\hline Sarcoidosis of the lung & $1(3.0)$ & 0 & 1 \\
\hline Visual loss & $1(3.0)$ & 1 & 0 \\
\hline \multicolumn{4}{|l|}{ Activity level } \\
\hline Low & $21(63.7)$ & 8 & 13 \\
\hline Medium & $11(33.3)$ & 5 & 6 \\
\hline High & $1(3.0)$ & 1 & 0 \\
\hline \multicolumn{4}{|l|}{ CIS-R study entry } \\
\hline Mild & $7(21.2)$ & 3 & 4 \\
\hline Moderate & $21(63.6)$ & 9 & 12 \\
\hline Severe & $5(15.2)$ & 3 & 2 \\
\hline \multicolumn{4}{|l|}{ Beck Depression Inventory score } \\
\hline Range & $15-57$ & $15-57$ & $18-45$ \\
\hline Mean & 29.6 & 31.7 & 33.8 \\
\hline \multicolumn{4}{|l|}{ Location of practice } \\
\hline Urban & $19(57.5)$ & 12 & 7 \\
\hline Suburban & $5(15.1)$ & 2 & 3 \\
\hline Rural & $9(27.2)$ & 4 & 5 \\
\hline
\end{tabular}

${ }^{a} \mathrm{n}(\%)$ unless otherwise indicated. CIS-R = Clinical Interview Schedule Revised. own homes, two in healthcare centres, and three on university premises. The interviews lasted between 30 and 120 minutes. A semi-structured topic guide was used to ensure consistency across the interviews (Box 1).

Following participant consent, the interviews were audiorecorded and transcribed verbatim.

\section{Data analysis}

Data collection and analysis proceeded in parallel, so that analysis of earlier interviews could inform the focus of later interviews. Data collection ended when data saturation had been reached, this being when no new themes were emerging from the data.

Transcripts were read and reread to gain familiarisation with the data; three of the researchers then met to discuss the transcripts and how they had interpreted and analysed what participants were saying and what insights and understanding the data gave.

Emerging themes were then identified and verified through discussion, and a coding frame based on these themes was developed. The same three members then independently coded transcripts and met to discuss their coding. This process led to existing codes being defined more clearly, and new codes being developed.

Transcripts were then coded using ATLAS.ti. Data pertaining to these codes were summarised in tables, using a framework approach, ${ }^{19}$ and verified by two researchers for consistency and comprehensiveness in exploring emerging themes. The meaning of the themes emerging from the coding of participants' accounts was often explicit, such that the evidence appeared overtly in the reasoning within the participants' responses. ${ }^{20}$ There was also implicit reasoning, in that the researchers were looking for the underlying logic in participants' accounts and using common sense to search for explanations.

Employing the framework approach enabled identification of what individual participants had said in relation to specific issues, and exploration of whether there appeared to be any link between, for example, how they viewed depression and the effectiveness of

\section{Box 1. Structure of topic guide.}

- Views of cause and impact of depression: is physical activity helpful for managing depression?

- $\quad$ intensity and form of activity; and

- mechanism of effect

Motivation and barriers to physical activity

- Acceptability of physical activity among other treatments 
physical activity as a treatment. It also enabled the researchers to compare what different participants had said in relation to the same issue, and thus to explore whether there were differences between the views expressed by males and females, and individuals of varying age, activity level at study entry, and level of depression. The researchers also sought to find contradictory or disconfirming evidence in relation to the emerging themes.

\section{RESULTS}

Most participants perceived physical activity to be an acceptable treatment for depression. The mechanisms by which physical activity could enhance mood were attributed to a number of subjective benefits including biochemical pathways, and providing a source of distraction from negative thoughts and a sense of purpose. Participants reporting a belief in biochemical mechanisms thought activity that 'raised the heartbeat' was most beneficial, while those reporting that exercise can act as a distraction from depression tended to state the benefits of less-aerobic activities, such as walking.

Many participants reported low motivation and a lack of confidence as barriers to undertaking physical activity. Many participants were taking antidepressant medication at the time of interview and some of these participants suggested that medication was helpful for initiating and maintaining activity.

Data pertaining to the themes are presented within four subheadings based on key areas explored during the interviews; within these key areas themes were identified through inductive and deductive analysis: (i) can physical activity help manage depression?; (ii) how does physical activity help manage depression?; (iii) the feasibility of assimilating physical activity into daily life; and (iv) views of physical activity compared with other treatments for depression. Where participants have been quoted, information is provided on their sex, age, treatment allocation, level of activity, and severity of depression.

\section{Can physical activity help manage depression?}

There was a general awareness among participants that physical activity could be an effective means of managing depression. This awareness was evident for most patients, across both arms of the study, sex, age range, activity levels, and classification of depression at study entry, and was informed anecdotally through the media and participants' own experiences:

'You read a lot things, and I remember reading that Ronnie O'Sullivan, the snooker player, he goes running as an antidote to the fact that he suffers from depression.' (male, 43 years, intervention, medium active, severe depression)
'I think it's really a no-brainer. I always feel energised and elevated after I have done something that's caused me hard work, my heart to beat faster. And usually when l've achieved something, you get a sense of euphoria. So I would have thought that exercise does help.' (female, 63 years, usual care, low active, severe depression)

\section{How does physical activity help manage depression?}

Participants described a number of subjective benefits to be gained from engaging in activity that may serve to enhance mood. These benefits included the perception of increasing mood enhancing chemicals in the brain, such as serotonin and endorphins, and providing a source of distraction from negative thoughts, and a sense of purpose:

'There's the physical wellbeing, feeling good, the emotional sense of challenge as I always push myself. The chemical high when you get back from a run and the emotional feel you get from actually pushing yourself into doing something.' (male, 35 years, usual care, low active, mild depression)

I know that if you increase the amount of movement and your activity then your serotonin level is going to kick in and it's going to make you feel better.' (female, 48 years, intervention, low active, moderate depression)

Many of the participants who had been classified as 'medium' or 'high' active participants at entry to the trial stated that they thought aerobic activities would be helpful for depression. These individuals usually cited a biochemical imbalance in the brain as a cause of depression, and it was apparent that they believed enhanced mood could be achieved through a biochemical pathway:

'I think it probably has to be a bit aerobic. Because I think that some of it is getting your blood supply working fast enough and getting the endorphins going, and I'm not sure that they work on very slow things.' (female, 65 years, usual care, high active, mild depression)

However, a few participants also considered lessaerobic activities as helpful for enhancing mood, due to having a relaxing or meditative quality:

'I don't know, for me the karate has that kind of meditational quality, an effect which is clearly not down to there being a kind of a high.' (male, 43 
years, intervention, medium active, severe depression)

Participants citing situational factors as a cause of depression were more likely to suggest that lowerintensity activities, such as walking, could be helpful as a means of distraction. Those reporting low levels of activity at study entry had a tendency to believe lowintensity activities could be helpful:

'I know how it [physical activity] can help distract me. And I switch then to positive thoughts, you know, positive thinking about much better things, so I know it works. I know when l've gone out walking with my friend, I'm concentrating on my surroundings which are great, I'm looking at different things, I'm not thinking about all the negative terrible things that I seem to have infested myself with. So I know it works, it makes you think much more positively.' (female, 63 years, usual care, low active, severe depression)

A number of participants also reported that they experienced the benefits of physical activity through the interaction of a number of factors. For example, some participants reported that engaging in physical activity could facilitate social interaction, regulate sleep cycles and eating behaviour, and control weight - a holistic experience that, in turn, could enhance selfconfidence and self-esteem:

I've lost half a stone without really trying, and that's very positive. Obviously the less weight your body carries, the more energy you have. So the less weight l've carried l've found myself able to keep going a lot longer and do more without the need to sit down for 10 minutes or rest for 10 minutes. And l'm sleeping better, which in turn, when I wake up in the morning you feel more optimistic. So it's benefits all the way around I think.' (female, 63 years, usual care, low active, severe depression)

'You're feeling better about yourself and I think that lifts the mood and then the sleep pattern usually, you know, it has a knock on effect, eating wise you, you feel more like eating and more regularly.' (male, 60 years, usual care, low active, moderate depression)

The benefits of engaging in physical activity could also be anticipated before engaging in an activity and could provide a sense of purpose that would lead to further positive actions:

I'm doing an activity that I'm satisfied with, that's fine, I'm going to get self-esteem from it, so therefore when l've made the decision to go and do it the process has already begun before the activity has started.' (male, 55 years, intervention, low active, moderate depression)

'When you are doing activity you are more pumped up, the adrenaline is going, the excitement is back, the pleasure is back. I'm a lot clearer [after physical activity] on what I want to do. I get more focused on things, menial tasks. Everything's working as it should be as far as I'm concerned.' (male, 44 years, intervention, medium active, mild depression)

\section{The feasibility of assimilating activity into daily life}

Many participants described both physical and mental symptoms of depression. The physical symptoms included lethargy and fatigue, while the emotional symptoms included low confidence. Such symptoms were described as hindering efforts towards increasing activity and were evident across the study arms, sex, age range, and all levels of activity and severity of depression:

'You feel as though you are walking through a bog in the fog, like you're dragging your limbs around - you need to get out of that stage in order to start doing something.' (male, 55 years, intervention, low active, moderate depression)

'You find yourself withdrawing until you find that, you find a comfortable place, like your home, and you just want to be safe in that place. And you feel that if you venture out of that through physical activity you feel more vulnerable, until you get to a point when you're beginning to come through the worst of it, and then physical activity is not so daunting, you feel more positive.' (female, 38 years, usual care, low active, moderate depression)

Although the positive aspects of activity were emphasised, some participants described negative aspects of attempting to engage in physical activity that could be demotivating, and barriers to being physically active. These negative aspects mainly related to ability and confidence and tended to be reported by female, low-active participants:

'I didn't enjoy indoor rock climbing at all. I have absolutely no upper body strength so it was - I didn't like it, it made me feel like I was inadequate.' (female, 24 years, usual care, low active, moderate depression) 


\section{Views of physical activity compared with other treatments for depression}

A few participants stated that the effectiveness of physical activity in managing depression would depend on the severity of depression and whether depression was thought to have a biochemical basis or to be due to situational factors:

'I suppose it's difficult because without sort of having antidepressants or counselling or whatever, it's difficult to know whether that [physical activity] would be enough on its own. I suppose it depends on the level of depression and issues that may be causing it.' (female, 40 years, usual care, low active, severe depression)

However, most participants stated a preference for physical activity over other treatments, particularly antidepressants, expressing a desire for some autonomy in the longer-term management of their depression, which they felt could be gained from engaging in physical activity:

I am increasing my confidence, physical activity, and some of these more complementary things need to take over from perhaps some traditional medication, you know. Because l've got perhaps 30 years to live, do you know what I mean? And I don't want to be considering taking long-term medication, you know.' (female, 48 years, intervention, low active, moderate depression)

However, one participant who was seeing a counsellor emphasised she felt there would still be a need for counselling, while another participant suggested that physical activity was not enough by itself, and a deeper level of understanding emotions was required to help with depression; both participants were in the usual-care arm of the trial and classified as being low active and with moderate depression:

'I find that seeing a counsellor is useful to getting things off your chest that maybe you wouldn't want to talk to your family about ... So I feel still being able to talk to somebody, I feel is useful for me. It may be the case of physical activity for other people is enough, but I'm not sure, my personal experience is that I couldn't do it without other help.' (female, 38 years, usual care, low active, moderate depression)

I know that physical activity is so helpful to how you feel and moving out of depression, or to help manage it, but it doesn't just go away by going off and jumping around for a couple of hours in an exercise class. You've got to learn to, sort of understand how things make you feel.' (female, 66 years, usual care, low active, moderate depression)

In addition, acknowledgment of the difficulties in engaging in physical activity, such as low motivation and confidence, meant that some participants viewed physical activity as an adjunct to medication, particularly for those experiencing more severe episodes of depression. Medication was seen to have a 'time and place', such that it could assist patients in initiating physical activity:

I think l've reached the stage with fluoxetine where it's kick-started the process [of engaging in activity]. I hope I have, I feel as though I have.' (male, 55 years, intervention, low active, moderate depression)

A few participants suggested that taking antidepressants could lead to dependence, although it was not clear whether they meant physical or emotional dependence:

'Yeah and I didn't want to [take tablets]. Because obviously I know a few people with depression and who are actually on antidepressants, and they've got it for quite a few years and it's hard to get off.' (male, 38 years, intervention, low active, moderate depression)

However, some saw medication as a more reliable and stable treatment in terms of adherence, due to the ease of taking a pill compared with the motivation and commitment required for engaging in physical activity:

'There's no excuse for you not being able to take them [tablets], whereas for exercise you have to be a bit more sporadic about it sometimes, there are other things that will consume your time.' (female, 24 years, usual care, low active, moderate depression)

Only two patients reported a risk of dependence on physical activity. Both suggested it could become an obsessive pursuit:

'You can almost become obsessive with trying to feel good all the time, so you end up exercising yourself, you know, like a lunatic, just trying to achieve that high all the time. So yeah I think you can go to the extreme.' (female, 48 years, intervention, low active, moderate depression)

'I became too obsessive, it was all or nothing approach and I would wear myself out.' (female, 
45 years, intervention, low active, mild depression)

\section{DISCUSSION}

\section{Summary of main findings}

The present study explored the views held by participants taking part in a trial to assess the effectiveness of physical activity for managing depression. However, the aim of the present article is not to evaluate the efficacy of the trial intervention, but rather to determine if participants felt that physical activity was as an acceptable treatment for depression.

Participants in the trial perceived physical activity as an acceptable treatment that could enhance mood for a wide range of individuals with depression. The mechanisms by which physical activity could enhance mood were attributed to a number of subjective benefits, including biochemical pathways, distraction from negative thoughts, and providing a sense of structure to daily life. Participants reported other benefits of engaging in activity, such as facilitating social interaction, weight loss, and regulation of sleep and eating patterns, which could lead to a holistic sense of wellbeing that in turn increased self-esteem.

However, the perceived cause of depression had a bearing on the extent that participants thought activity might be helpful, suggesting it was less beneficial if depression was believed to be a function of situational factors as opposed to being of biochemical origin. In addition, participants who thought depression was due to a biochemical imbalance rather than to situational factors tended to state that physical activity had to be aerobic in nature, while those believing their depression was related to situational or adverse life events tended to report the benefits of less-intense aerobic activities, such as walking. Low-intensity activity, such as walking, may be helpful as a means of distraction, and it is postulated that this may be helpful with more ruminative responses to depression. ${ }^{21}$

\section{Strengths and limitations of the study}

This is the first study the authors are aware of that has examined patients' views of physical activity for depression in primary care that is not in the context of exercise referral schemes. The content of the patient information sheets may have had a priming effect for participants. However, such priming may not necessarily influence their perceptions of how activity might be helpful, their views on the cause and impact of depression, their ability to undertake activity, and its acceptability among other treatments. Interviews were largely conducted in participants' homes with a researcher who was independent of the trial in which they participated. This may have helped to elicit a high degree of candour and openness from participants.

The generalisability of the study findings, however, may be limited, as participants were primarily white
British, and depression is known to affect individuals of all ethnic backgrounds who may have different views. ${ }^{8}$ However, the authors believe that a range of socioeconomic backgrounds were represented in the sample, although the majority of responders had attained advanced educational qualifications.

In addition, sampling participants from an activity trial may have meant a select group of participants were interviewed, who held particularly positive views about physical activity as a treatment for depression or were primed by the information provided to them before consenting to participate in the main trial. Furthermore, the interviews were conducted 4 months into the trial, so participants in the intervention arm, in particular, may have altered perceptions of physical activity. However, no differences were detected in views between participants in the usual-care and intervention arms with regard to the acceptability and efficacy of physical activity.

\section{Comparison with existing literature}

There was some evidence that participants may prefer the 'self-help' aspect of engaging in physical activity as opposed to the passive nature of taking antidepressant medication, despite the fact that many were currently being treated with antidepressant medication. Previous work has shown that physical activity is primarily seen by patients as an alternative coping mechanism - a credible next step after prior stabilisation with medication. ${ }^{14}$ In addition, physical activity may be viewed in a 'self-regulatory' way, as a means of providing temporary relief from symptoms, rather than a cure for depression. ${ }^{15}$ However, some participants in the present study suggested that medication had enabled them to initiate and maintain physical activity, as it helped them to overcome barriers to activity, such as low levels of motivation and confidence which may be a particular problem in patients with depression. ${ }^{7}$ It was with reference to these barriers that participants felt that it would be easier to take a pill than participate in regular activity, implying a recognition of problems in overcoming, initiating, and maintaining physical activity as a treatment.

\section{Implications for clinical practice and future research}

NICE guidance states that GPs should consider offering structured group-based physical activity programmes to patients with depression. ${ }^{2}$ However, despite evidence of its effectiveness, ${ }^{5}$ prescribing exercise may not be a sufficient means to encourage and maintain physical activity, due to the individual nature of patients' views of depression and physical activity as a potential treatment. Johnston et al highlighted the need for physicians to have a greater awareness of the extent to which their goals for the 
management of depression are perceived as relevant or achievable by patients. ${ }^{22}$ The present findings demonstrate that a wide range of individuals feel they would benefit from physical activity as a treatment, and the type and level of activity perceived to be beneficial varied between individuals and circumstances. Such findings resonate with work by Johnston et al, which supports the idea that GPs should explore patients' perceptions of physical activity as a treatment before recommending physical activity either alone or as an adjunct treatment. ${ }^{22}$

The findings of the present study also have implications for 'stepped care', outlined in NICE guidance. The principal tenet of 'stepped care' is based on patient-led self-help with limited intervention from professionals. ${ }^{23}$ Adopting stepped care would suggest that physical activity is promoted prior to approaches based on antidepressant medication. However, the present findings and those of Faulkner and Biddle ${ }^{14}$ suggest that the order of stepped care is evaluated on an individual basis to reflect patients' perceptions of a treatment such as physical activity, and their ability to engage with it - particularly as patients lacking motivation to undertake physical activity may benefit from antidepressant medication. In some cases it may be necessary to prescribe antidepressant medication prior to the recommendation of physical activity.

The issues relating to non-adherence to activity may be alleviated by developing and delivering individually tailored physical activity interventions, such as that of the TREAD trial; however, its efficacy over and above usual care with antidepressant medication is yet to be determined. Future research should consider the ability of patients with coexisting physical health problems to engage with physical activity, ${ }^{24}$ thus reinforcing the need for individual tailoring of any such advice to the physical and psychological status of the potential patient. In addition, it is important to explore the views of GPs with regard to recommending activity to their patients with depression, and to evaluate the provision of tailored interventions for managing depression in primary care.

\section{Funding body}

Health Technology Assessment (HTA) grant, Project no: 03/45/07.

\section{Ethics committee}

Ethical approval to interview patients was obtained from West Midlands Multicentre Research Ethics Committee: MREC no: 05/MREC07/42. All participants gave written informed consent prior to interviews.

\section{Competing interests}

The authors have stated that there are none.

\section{Acknowledgements}

We would like to thank all the participants for sharing their views, and are grateful for the contribution of primary care centres participating in the trial from which study participants were recruited. We are also very grateful to
Melanie Chalder (TREAD trial coordinator, Bristol) and Sarah Dawkins (research administrator, Exeter) for their assistance with participant recruitment and liaison).

\section{Discuss this article}

Contribute and read comments about this article on the Discussion Forum: http://www.rcgp.org.uk/bjgp-discuss

\section{REFERENCES}

1. Mead GE, Morley W, Campbell P, et al. Exercise for depression. Cochrane Database Syst Rev 2009; (3): CD004366.

2. National Institute for Health and Clinical Excellence. Depression: management of depression in primary and secondary care. NICE clinical guideline 90. London: National Institute for Health and Clinical Excellence, 2009.

3. Department of Health. Exercise referral systems: a National Quality Assurance Framework. London: Department of Health, 2001. http://www.dh.gov.uk/assetRoot/04/07/90/09/04079009.pdf (accessed 25 October 2010).

4. Mental Health Foundation. Moving on up. London: Mental Health Foundation, 2009. http://www.mentalhealth.org.uk/publications/ ?entryid5=73298\&p=3\&char=M (accessed 25 October 2010).

5. Williams NH, Hendry M, France B, et al. Effectiveness of exercise referall schemes to promote physical activity in adults: systematic review. Br J Gen Pract 2007; 57(545): 979-986.

6. Donaghy M, Taylor AH. Current controversies: should practitioners promote physical activity as a treatment for depression? JR Coll Physicians Edinb 2010; 40: 132-135.

7. Seime RJ, Vickers KS. The challenges of treating depression with exercise: from evidence to practice. Clin Psychol Sci Pract 2006; 13: 194-197.

8. World Health Organization. The World Health Report 2001: mental health: new understanding, new hope. Geneva: World Health Organization, 2001.

9. Lester $\mathrm{H}$, Howe A. Depression in primary care: three key challenges. Postgrad Med J 2008; 84(996): 545-548.

10. Gunnell D, Ashby D. Antidepressants and suicide: what is the balance of benefit and risk? BMJ 2004; 329(7456): 34-38.

11. Hollinghurst S, Kessler D, Peters T, Gunnell D. Opportunity cost of antidepressant prescribing in England: analysis of routine data. $B M J$ 2005; 330(7498): 999-1000.

12. Hyde J, Calnan M, Prior L, et al. A qualitative study exploring how GPs decide to prescribe anti-depressants. Br J Gen Pract 2005; 55(519): 755-762.

13. Van Geffen ECG, Gardardsottir H, Van Hulten R, et al. Initiation of antidepressant therapy: do patients follow the GP's prescription? $B r \mathrm{~J}$ Gen Pract 2009; 59(559): 81-87.

14. Faulkner G, Biddle SJH. Exercise and depression: considering variability and contextuality. J Sports Exerc Psychol 2004; 26: 3-18.

15. White KT. Why does physical activity alleviate depression? Identifying potential mediators and understanding the process of change. PhD thesis. Southampton: University of Southampton, 2008.

16. Baxter H, Winder R, Chalder M, et al. Physical activity as a treatment for depression: the TREAD randomised trial protocol. Trials 2010, 11: 105. doi:10.1186/1745-6215-11-105.

17. Lewis G, Pelosi AJ. The manual of CIS-R. London: Institute of Psychiatry, 1992.

18. Beck AT, Ward CH, Mendelsohn M, et al. An inventory for measuring depression. Arch Gen Psychiatry 1961, 4: 561-571.

19. Ritchie J, Lewis J. Qualitative research practice: a guide for social scientists and researchers. London: Sage Publications Ltd, 2003.

20. Lofland J, Lofland LH. Analyzing social settings. 3rd edn. Belmont, CA: Wadsworth, 1995.

21. Broderick. Mindfulness and coping with dysphoric mood: Contrasts with rumination and distraction. Cognit Ther Res 2005; 29(5): 501-510.

22. Johnston O, Kumar S, Kendall K, et al. Qualitative study of depression management in primary care: GP and patient goals and the value of listening. Br J Gen Pract 2007; 57(544): el-e14.

23. Davison GC. Stepped care: doing more with less? J Consult Clin Psychol 2000; 68(4): 580-585.

24. Pilling S, Anderson I, Goldberg D, et al. Depression in adults, including those with chronic physical health problem: summary of NICE guidance. BMJ 2009; 359: b4108 